\title{
Internal Flow Condition between Front and Rear Rotor of Contra-Rotating Small-Sized Axial Fan at Low Flow Rate
}

\author{
Toru Shigemitsu', Kensuke Tanaka², Katsuhiko Hirosawa², Keisuke Miyazaki \\ ${ }^{1}$ Institute of Science and Technology, Tokushima University, Tokushima, Japan \\ ${ }^{2}$ Graduate School of Advanced Technology and Science, Tokushima University, Tokushima, Japan \\ Email: t-shige@tokushima-u.ac.jp
}

How to cite this paper: Shigemitsu, T., Tanaka, K., Hirosawa, K. and Miyazaki, K. (2017) Internal Flow Condition between Front and Rear Rotor of Contra-Rotating Small-Sized Axial Fan at Low Flow Rate. Open Journal of Fluid Dynamics, 7, 709-723. https://doi.org/10.4236/ojfd.2017.74046

Received: November 29, 2017

Accepted: December 26, 2017

Published: December 29, 2017

Copyright $\odot 2017$ by authors and Scientific Research Publishing Inc. This work is licensed under the Creative Commons Attribution International License (CC BY 4.0).

http://creativecommons.org/licenses/by/4.0/

\begin{abstract}
Contra-rotating small-sized fans are used as cooling fans for electric equipment. The internal flow condition between the front and rear rotors of the contra-rotating small-sized fan is not known well especially at the low flow rate. Furthermore, the blade row distance between the front and rear rotors is an important parameter for the contra-rotating small-sized fan and its influence on the internal flow condition is not clarified at the low flow rate. Therefore, the internal flow condition of the contra-rotating small-sized fan at the low flow rate is investigated by the numerical analysis in this research. The numerical analysis results are validated by comparing the fan static pressure curves of the numerical results to the experimental results. The internal flow condition at the low flow rate is clarified using the numerical models of the different blade row distance $L=10 \mathrm{~mm}$ and $30 \mathrm{~mm}$. In the present paper, pressure fluctuations phase locked each front and rear rotor's rotation are shown and the influences of the wake and the potential interference are discussed by the unsteady numerical analysis results at the low flow rate.
\end{abstract}

\section{Keywords}

Small-Sized Axial Fan, Contra-Rotating Rotors, Wake, Potential Interference, Numerical Analysis

\section{Introduction}

Small-sized axial fans are used as cooling fans for electric equipment i.e. laptop, desk top computers and servers. The electrical power used for the cooling of the IT devices for data centers is huge the same as the electric power used for the IT devices itself. There is a strong demand for higher pressure and larger flow rate 
of fans according to the increase of quantity of heat from electric devices. Therefore, the contra-rotating rotors are adopted for the small-sized axial fans to increase the performance. The lower rotational speed design [1] and advantages on the performance of the contra-rotating fans and pumps were verified by experimental results [2] [3]. In the case of contra-rotating rotors, the axial space becomes larger than conventional small-sized axial fans. However, it is adequate choice to apply the contra-rotating rotors for small-sized fan because the axial space can be ensured in electrical devices as compared to that of the radial space.

In the case of the contra-rotating rotors, it is necessary to design the rear rotor considering the unsteady circumferential velocity distributions at the outlet of the front rotor [4]. Further, the fan noise becomes larger than the conventional rotor stator type fan because of the interaction between the front and rear rotors. Then, the passive noise reduction with the perforated blade was proposed for the contra-rotating fan [5]. It is important to clarify the influence of the wake from the front rotor to the rear rotor and the potential interaction between the front and rear rotors to increase the performance and to reduce the fan noise [6]. The blade row distance between the front and rear rotors is a key parameter to consider the wake and potential interaction for the contra-rotating fan. The influence of the blade row distance between the front and rear rotors and pressure fluctuations on the casing wall were investigated for the counter rotating fan with fan diameter $D=375 \mathrm{~mm}$ [3] [7]. On the other hand, the conventional design method and the theory for the turbomachinery should be modified for small-sized axial fans because small-sized axial fans applied to electrical devices belong to extremely small size field in the turbomachinery [8]. Therefore, there is the strong demand to establish the design method for small-sized axial fans based on the internal flow. Then, the internal flow condition was investigated using the numerical models having different blade row distance $L=10 \mathrm{~mm}$ and $30 \mathrm{~mm}$ at the design flow rate [9]. Regarding the conventional axial flow fan, the relative velocity vectors and streamlines at off-design flow rate points with the deep stall condition were computed using the downstream flow resistance method [10]. Unsteady flow in axial skewed fans was measured at off-design conditions using a hot-wire anemometer, to investigate the effect of sweep on the performance and flow condition [11]. On the other hand, the internal flow condition of the contra-rotating axial flow fan at off-design flow rate point is not known well. Then, the internal flow condition at partial flow rate was investigated by a pitot-tube and numerical analysis, and it was clarified that the back flow associated with the tip leakage flow occurred at the inlet tip of both front and rear rotors [12]. It is difficult to measure the internal flow condition at low flow rate and there is no literature related to the internal flow condition of the contra-rotating small sized axial fan at low flow rate. Therefore, the internal flow condition at the low flow rate was investigated by the numerical analysis.

In the present paper, the performance curves of the contra-rotating small-sized axial fan with $100 \mathrm{~mm}$ diameter are compared with the unsteady numerical 
analysis results to verify the validity of the unsteady numerical analysis results. After that, pressure fluctuations phase locked each front and rear rotor's rotation are shown and the influences of the wake and the potential interference at the low flow rate ( $10 \%$ of the design flow rate) are discussed by the unsteady numerical analysis results.

\section{Experimental Apparatus and Method}

The geometry of the rotor and primary dimensions of the contra-rotating small-sized axial fan (RRtype) are shown in Figure 1 and Table 1, respectively. The hub tip ratio was $D_{h} / D_{t}=45 \mathrm{~mm} / 98 \mathrm{~mm}$, tip clearance was $c=1 \mathrm{~mm}$ and the design flow rate was $Q_{d}=0.016 \mathrm{~m}^{3} / \mathrm{s}$. Fan static pressure at the design point was $P_{d R R}=14.7 \mathrm{~Pa}$ for RRtype with the same fan static pressure of each front and rear rotor. The rotational speed of the front and rear rotors for RRtype was $N_{f}=$ $N_{r}=1780 \mathrm{~min}^{-1}$. This fan was designed with the free vortex design. In this research, an airfoil blade was used because there was a report that mentioned an advantage of the airfoil blade for the small-sized axial fan [13], however, a circular-arc blade was generally used for small-sized axial fans. Figure 2 show the schematic diagram and picture of the experimental apparatus for RRtype.

The experimental apparatus was designed based on the Japanese Industrial

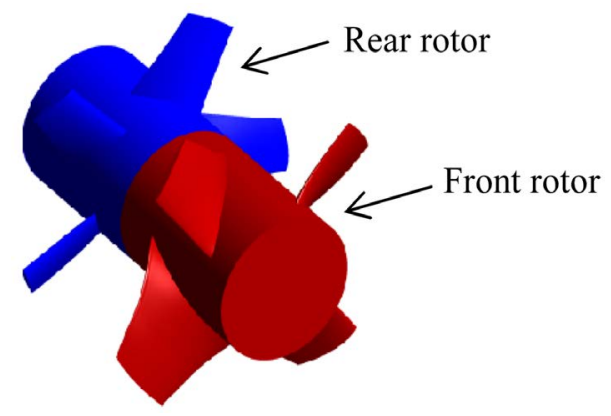

Figure 1. Contra-rotating small-sized axial fan (RRtype).

Table 1. Primary dimensions of RRtype.

\begin{tabular}{|c|c|c|c|c|}
\hline & \multirow{2}{*}{ Diameter $[\mathrm{mm}]$} & $\mathrm{Hub}$ & Mid & Tip \\
\hline & & 45 & 72 & 98 \\
\hline \multirow{4}{*}{ Front rotor } & Blade number & & 4 & \\
\hline & Blade profile & & NACA4409 & \\
\hline & Solidity & 1.196 & 0.496 & 0.290 \\
\hline & Stagger angle & $44.7^{\circ}$ & $61.1^{\circ}$ & $68.2^{\circ}$ \\
\hline \multirow{4}{*}{ Rear rotor } & Blade number & & 5 & \\
\hline & Blade profile & & NACA4412 & \\
\hline & Solidity & 0.910 & 0.447 & 0.288 \\
\hline & Stagger angle & $56.7^{\circ}$ & $64.5^{\circ}$ & $69.6^{\circ}$ \\
\hline
\end{tabular}




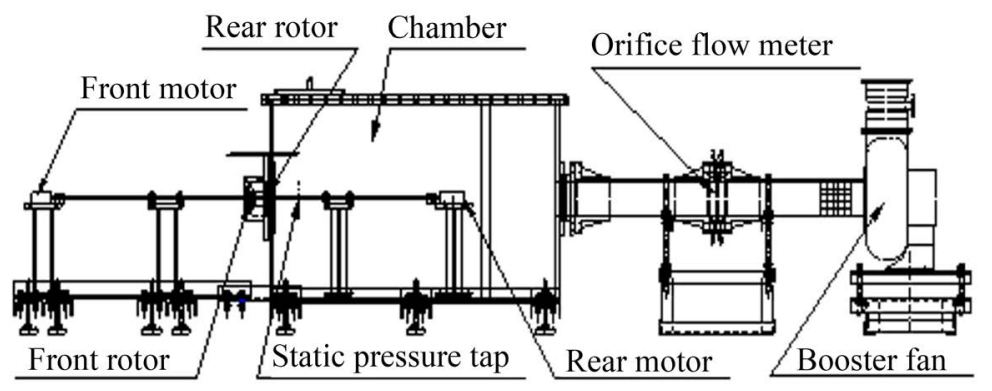

(a)

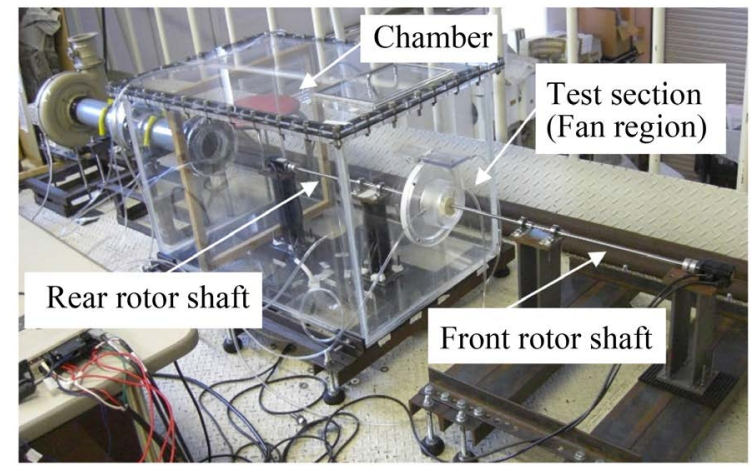

(b)

Figure 2. Experimental apparatus. (a) Schematic diagram of experimental apparatus; (b) Picture of experimental apparatus.

Standard (JIS B 8330 Testing methods for turbo-fans) and the air blown in the test section passed the rotor, chamber, measurement duct and booster fan and blew out in the ambient atmosphere. The static pressure taps were installed at the casing wall between the front and rear rotors and the chamber wall $80 \mathrm{~mm}$ downstream of the rotors. The static pressure was measured by the manometer (OKANO WORKS, LTD DMP201N12). Then, the fan static pressure $\left(P_{s}\right)$ was evaluated by the pressure difference between static pressure taps downstream of the rotor installed at the chamber and ambient air. The fan static pressure of each front and rear rotor was also evaluated to investigate pressure curves of each front and rear rotor in wide flow rates range. Each front and rear rotor was connected to the respective driving shaft and rotated by the servo motor (YASUKAWA SGMJV-A5ADA21 for the front rotor and SGMJV-01ADA21 for the rear rotor).Further, the rotational speed was controlled by the servo motor pack (YASKAWA SGDV-R70A01A for the front rotor and YASKAWA SGDU-R90A01A for the rear rotor). The flow rates were measured by an orifice meter set at the measurement duct and the static pressure difference of the orifice was measured by the manometer (OKANO WORKS, LTD DMP201N12 and DMP202N12). The pressure curves of RRtype from the cutoff flow rate to large flow rate were investigated in the experiment with the constant rotational speed $N_{f}=N_{r}=1780 \mathrm{~min}^{-1}$. In this research, the performance test was conducted with two different blade row distances $L=10 \mathrm{~mm}$ (non-dimensional axial distance 
divided by front rotor tip chord length $\left.L / I_{F R}=0.42\right), 30 \mathrm{~mm}\left(L / I_{F R}=1.27\right) . L$ was defined as the axial distance from the trailing edge of the front rotor to the leading edge of the rear rotor at the hub.

\section{Numerical Analysis Conditions}

The commercial software ANSYS-CFX 14.5 was used to investigate the flow condition between the front and rear rotors of the contra-rotating small-sized axial fan which couldn't be measured by the experiment. In the numerical analysis, the numerical model which was almost the same with the experimental apparatus was used. The unsteady numerical analysis was conducted using the three dimensional model. The numerical analysis grids used for the numerical analysis are shown in Figure 3. To simplify the numerical analysis, the servo motors and the shafts of the servo motors are removed in the numerical analysis model. The numerical domains comprised the inlet, rotor, chamber and outlet duct regions. The numerical grid elements were 617,090 for the inlet region, $1,479,336$ for the chamber region and 237,628 for the outlet duct region. The numerical grid elements of the rotor region were set to increase with the increase of the blade row distance, so the numerical grid elements for the rotor region were $5,542,038$ for $L=10 \mathrm{~mm}$ and $6,038,826$ for $L=30 \mathrm{~mm}$. The tip clearance kept $1 \mathrm{~mm}$ as the same with the experimental apparatus in the numerical analysis and the number of elements from the blade tip to the casing was 7 . At the inlet

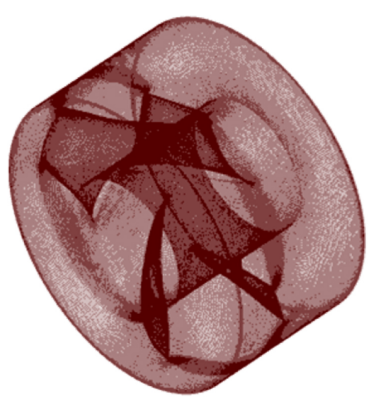

(a)

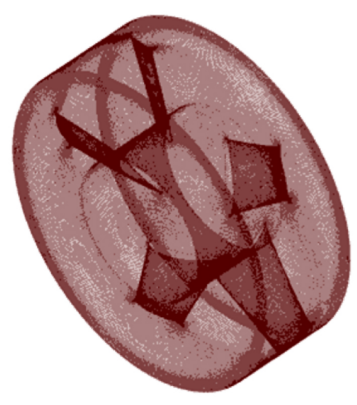

(b)

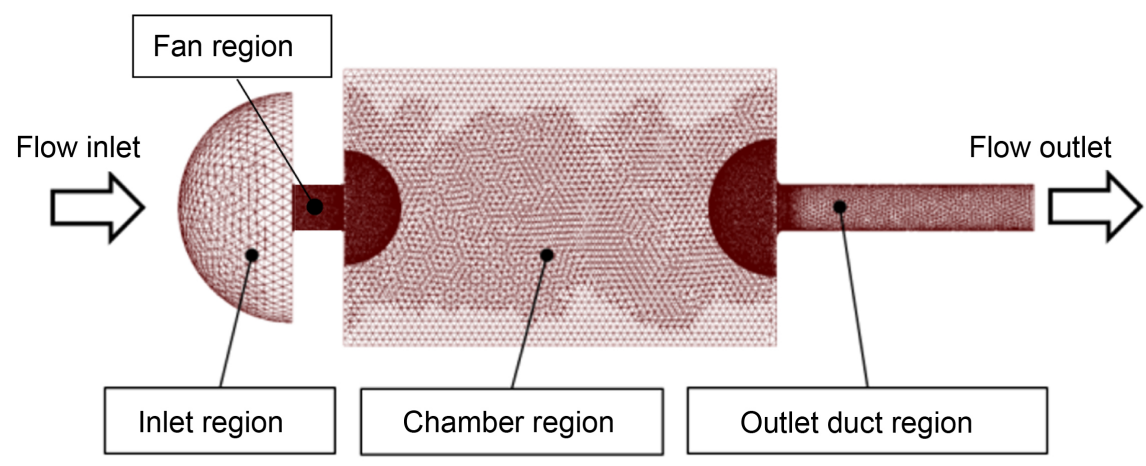

(c)

Figure 3. Numerical analysis grids. (a) Front rotor; (b) Rear rotor; (c) Whole domains. 
boundary, the uniform velocity was given and the constant pressure was given at the outlet boundary condition. Each front and rear rotor region was simulated on the relative reference frame considering the rotation of each rotor. Non-slip wall condition was used for the shroud and hub surfaces. In the rotational regions, the shroud was set to rotate in the opposite direction of the rotor to make the shroud stationary in the absolute coordinate system. The coupling between the front and rear rotors was conducted by the sliding mesh (Transient Rotor Stator Model). SST (Shear Stress Transport) turbulence model suitable for the estimation of the separation was used because the test fan having the complex flow with the separation near the wall. "Automatic" near wall treatment recommended for the SST model was used to allow for a smooth shift from a low-Reynolds number form to a wall function formulation. The unsteady numerical analysis was conducted at five flow rates $\left(0.1 Q_{\phi}, 0.6 Q_{\phi}, 0.9 Q_{\phi} 1.0 Q_{d}\right.$ and $1.2 Q_{d}$ ). The time step number per one rotor rotation was 180 and the time step was $t=1.8727 \times 10^{-4} \mathrm{~s}$ (corresponding $2^{\circ}$ of the rotor rotation). The data of one rotor rotation were obtained after 6 rotor rotations in unsteady numerical analysis. The convergence criteria at each time step were set as the residual less than $1.0 \times 10^{-4}$. The computational time for the unsteady numerical analysis was 10 14 days depending on the flow rate.

\section{Experimental and Numerical Analysis Results}

\subsection{Performance Curve of RRtype}

Fan static pressure curves of each blade row distance $(L=10,30 \mathrm{~mm})$ for RRtype, front rotor and rear rotor obtained by the experiment are shown in Figure 4. In Figure 4, the fan static pressure obtained by the numerical analysis is also given to compare with the experimental results. In the unsteady numerical analysis, the static pressure was obtained at the same point in the experiment and 180 static pressure data of one rotor rotation were averaged. The vertical axis and the horizontal axis in Figure 4 show the fan static pressure $P_{s}$ and the flow rate $Q$. It was found from Figure 4 that in both blade row distance $L=10$ $\mathrm{mm}$ and $30 \mathrm{~mm}$, the fan static pressure of RRtype linearly increased as the flow rate decreased, and the pressure curve of RRtype showed the stable negative curve. Further, the fan static pressure of the front rotor also showed the same tendency of RRtype, but the fan static pressure of the rear rotor represented a different tendency from the fan static pressure of RRtype in the partial flow rates. And the slight positive slope of the pressure curve was confirmed in the low flow rates for the rear rotor. The fan static pressure of $L=10 \mathrm{~mm}\left(P_{s}=11.47\right.$ Pa) was larger than that of $L=30 \mathrm{~mm}\left(P_{s}=11.45 \mathrm{~Pa}\right)$ by $\Delta P_{s}=0.02 \mathrm{~Pa}$ at the design flow rate $Q_{d}=0.016 \mathrm{~m}^{3} / \mathrm{s}$ in the experiment. On the other hand, the fan static pressure of $L=10 \mathrm{~mm}\left(P_{s}=26.87 \mathrm{~Pa}\right)$ was smaller than that of $L=30 \mathrm{~mm}$ $\left(P_{s}=27.50 \mathrm{~Pa}\right)$ by $\Delta P_{s}=0.63 \mathrm{~Pa}$ at the low flow rate $0.1 Q_{d}$. The influence of the blade row distance in the range of $L=10-30 \mathrm{~mm}$ on the fan static pressure was small, but there is a tendency that the fan static pressure of $L=30 \mathrm{~mm}$ became 


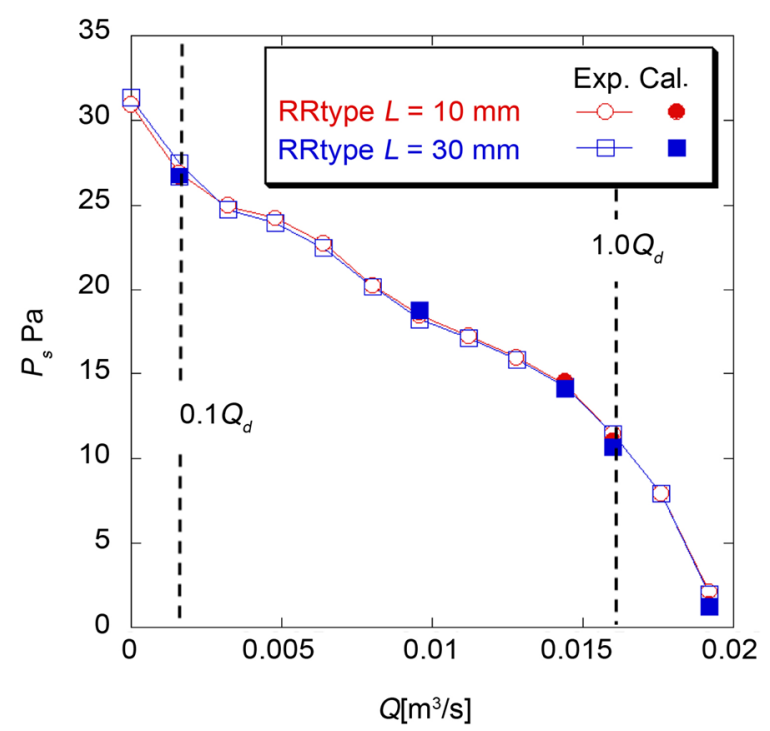

(a)

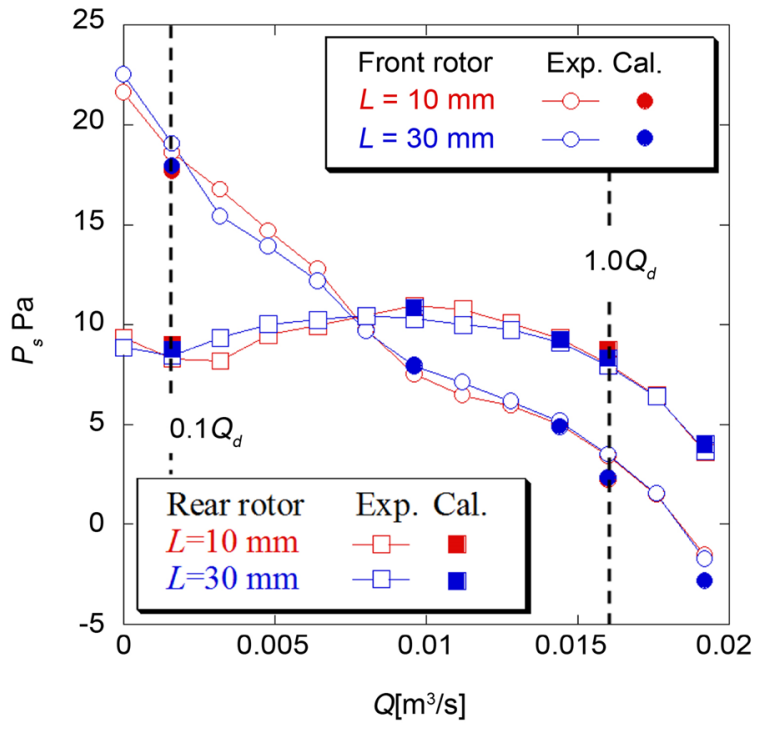

(b)

Figure 4. Fan static pressure of RRtype. (a) RRtype (Total);

(b) Each front and rear rotor.

large compared to that of $L=10 \mathrm{~mm}$ at the low flow rate. This characteristic could be confirmed in the numerical analysis results too.

The numerical analysis results of the fan static pressure could predict the experimental results accurately and capture the tendency of the performance curves of the experimental results. It was difficult to measure the efficiency of this small fan. Therefore, the efficiency of the test fan was investigated by the numerical analysis results. The static pressure efficiency was obtained by the following equation,

$$
\eta_{s}=\frac{P_{s} Q}{L_{s}}
$$


where $L_{s}$ is the shaft power obtained by the torque $T$ and angular velocity of the numerical analysis. The static pressure efficiency of RRtype for $L=10 \mathrm{~mm}$ and $30 \mathrm{~mm}$ is shown in Figure 5. The maximum static pressure efficiency of $L=10$ $\mathrm{mm}(\eta=58.0 \%)$ was larger than that of $L=30 \mathrm{~mm}(\eta=57.0 \%)$ by $1.0 \%$. On the other hand, the static pressure efficiency of $L=10 \mathrm{~mm}(\eta=11.2 \%)$ was smaller than that of $L=30 \mathrm{~mm}(\eta=11.3 \%)$ at the low flow rate $0.1 Q_{d}$. In general, the fan static pressure and static pressure efficiency of the contra-rotating axial fan decrease with the increase of the blade row distance. On the contrary, the performance increased or became almost the same with increase of the blade row distance from $L=10 \mathrm{~mm}$ to $L=30 \mathrm{~mm}$ at the low flow rate $0.1 Q_{d \cdot}$. This phenomenon is interesting, so the internal flow condition at the low flow rate $0.1 Q_{d}$ was investigated by the unsteady numerical analysis results.

\subsection{Internal Flow Condition between the Front and Rear Rotors at Low Flow Rate $0.1 Q_{d}(L=10,30 \mathrm{~mm})$}

The fan static pressure kept almost constant by the blade row distance between the front and rear rotors was $L=30 \mathrm{~mm}$. The difference of the fan static pressure between $L=10 \mathrm{~mm}$ and $L=30 \mathrm{~mm}$ were $\Delta P_{s}=0.02$ (Exp.), 0.4(Cal) Pa at the design flow rate $Q_{d}=0.016 \mathrm{~m}^{3} / \mathrm{s}$ and $\Delta P_{s}=-0.63$ (Exp.), $-0.02(\mathrm{Cal}) \mathrm{Pa}$ at the low flow rate $0.1 Q_{d}$. In the experimental and numerical analysis results, the fan static pressure of $L=10 \mathrm{~mm}$ is higher than that of $L=30 \mathrm{~mm}$ at the design flow rate $Q_{d}=0.016 \mathrm{~m}^{3} / \mathrm{s}$. However, the fan static pressure of $L=30 \mathrm{~mm}$ was approximately equal to or higher than that of $L=10 \mathrm{~mm}$ at the low flow rate $0.1 Q_{d}$ unlike at the design flow rate $Q_{d}=0.016 \mathrm{~m}^{3} / \mathrm{s}$. In this section, focused on this point, pressure fluctuations around the rotor phase locked each front and rear rotor's rotation are shown and the influences of the wake and the potential interference are discussed by the unsteady numerical analysis results. The sampling points for

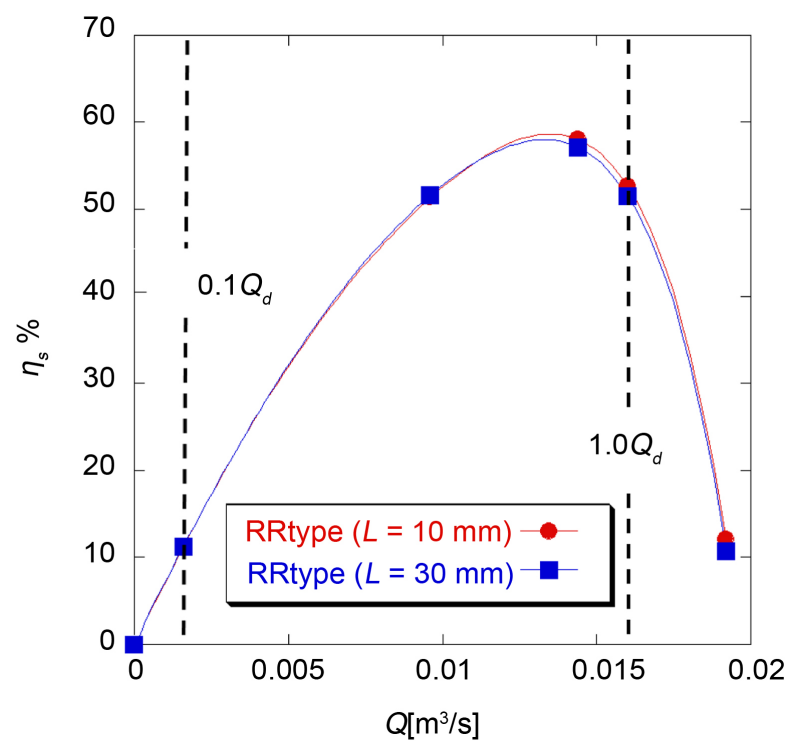

Figure 5. Static pressure efficiency of RRtype. 
the pressure fluctuations and the definition of the relative position of the front and rear rotors are shown in Figure 6. The respective data were acquired at 0.1 $\mathrm{mm}$ downstream from the trailing edge (TE) of the front rotor and $0.1 \mathrm{~mm}$ upstream from the leading edge (LE) of the rear rotor. The rotational angles of the front and rear rotors are shown as $\theta_{f} \theta_{r}$ respectively. The rotational angles were defined as the circumferential angles of the $25 \%$ chord length position of each front and rear rotor from the arbitrary meridional plane. The relative angle of the front and rear rotors $\theta_{f r}$ are defined as the following equation,

$$
\theta_{f r}=\theta_{f}+\theta_{r}
$$

where the default of the relative angle $\theta_{f r}$ is $\theta_{f r}=0$ deg. $\left(\theta_{f}=\theta_{r}=0\right.$ deg.). The rotational direction of the angle $\theta_{f} \theta_{r}$ correspond the rotational direction of the front and rear rotors, respectively. The pressure fluctuations of the trailing edge of the front rotor and the leading edge of the rear rotor at the low flow rate $0.1 Q_{d}$ from the numerical analysis results are shown in Figure 7 and Figure 8. The vertical axis is the static pressure fluctuation $\Delta P$ and the horizontal axis is the relative angle of the front and rear rotors $\theta_{f r}$ in Figure 7 and Figure 8. Further, the symbol $r / r_{c}$ shows non-dimensional radius divided by the radius at the casing; $r / r_{c}=0.45$ and $r / r_{c}=1.0$ correspond the hub and the casing. Because the pressure in Figure 7 was phase locked the front rotor rotation, the potential interference from the rear rotor became clear, if the unsteady pressure fluctuations from the front rotor were small; the periodic fluctuations with five peaks corresponding to the rear rotor blade number $Z_{R}=5$ was supposed to be observed in Figure 7. In Figure 7, contrary to our expectations, respective curves (red, blue and green curves) at each radial position are not periodic for both $L=10$ $\mathrm{mm}$ and $L=30 \mathrm{~mm}$. This tendency was confirmed in Figure 8 phase locked the rear rotor rotation; There was no periodic pressure fluctuation related to the front rotor wake and potential interference with four peaks corresponding to the front rotor blade number $Z_{F}=4$. The characteristic difference of the pressure fluctuation for $L=10 \mathrm{~mm}$ and $L=30 \mathrm{~mm}$ was not observed in Figure 7 and

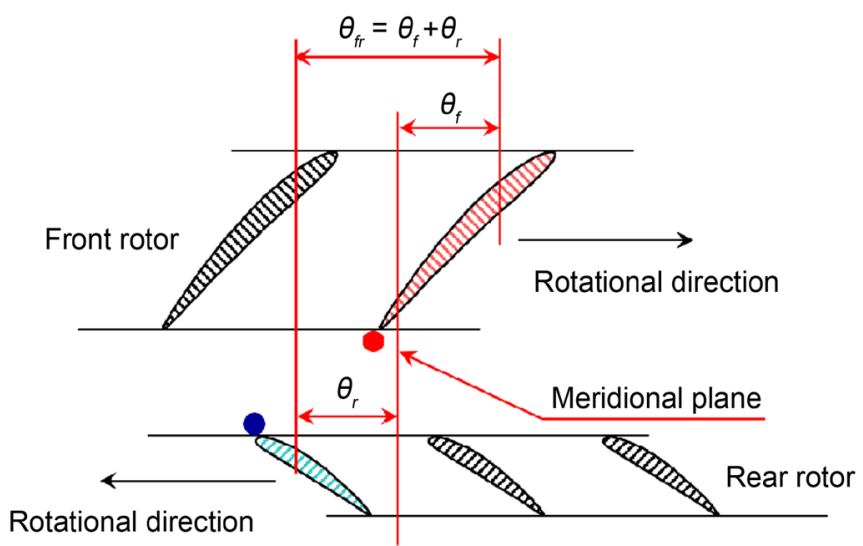

Figure 6. Sampling points and relative position of front and rear rotors. 


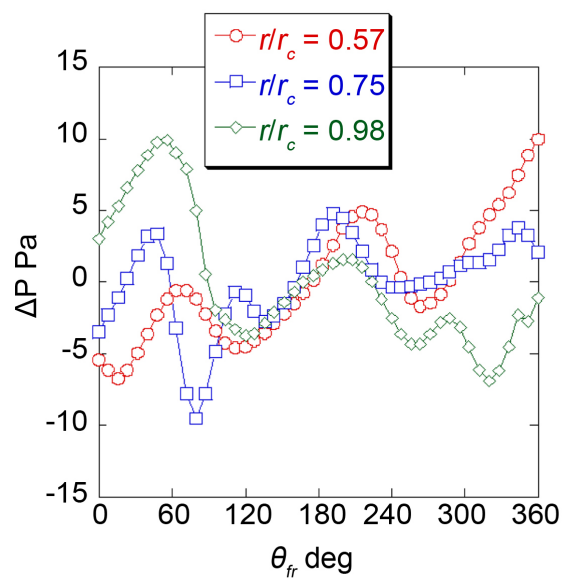

(a)

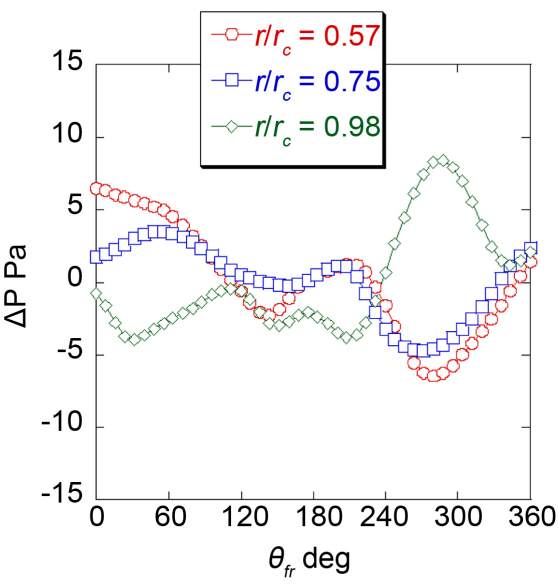

(b)

Figure 7. Pressure fluctuations of the trailing edge of front rotor. (a) $L=10 \mathrm{~mm}$; (b) $L=$ $30 \mathrm{~mm}$.
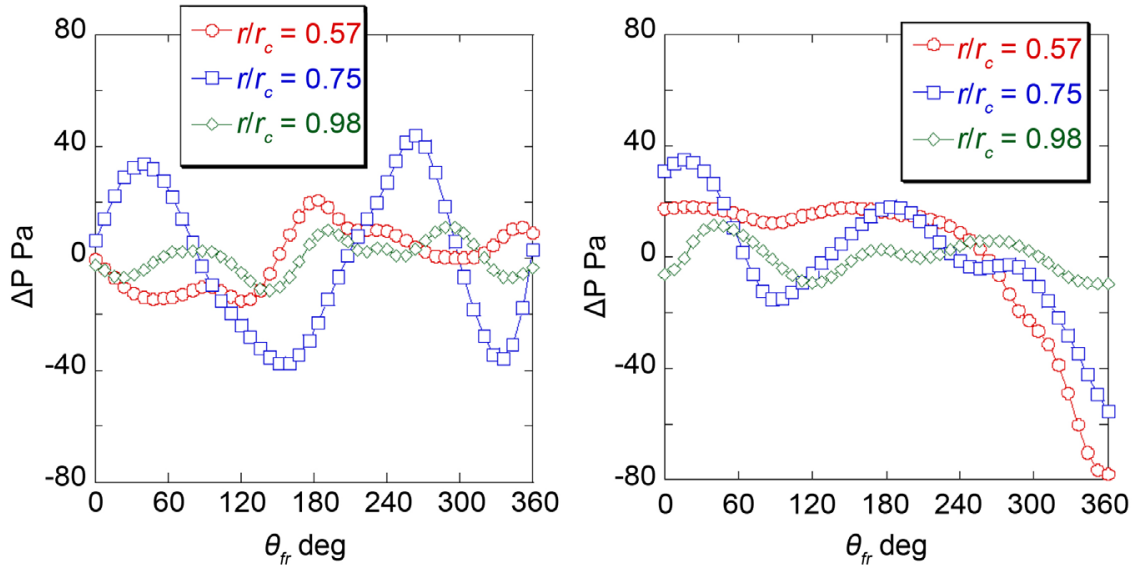

Figure 8. Pressure fluctuations of the leading edge of rear rotor. (a) $L=10 \mathrm{~mm}$; (b) $L=$ $30 \mathrm{~mm}$.

Figure 8. These results lead to think that the flow condition and the potential interference between the front and rear rotors at the low flow rate $0.1 Q_{d}$ are significantly complex. Therefore, we investigated the wake and the potential interference between the front and rear rotors at the low flow rate $0.1 Q_{d}$ from the numerical analysis results.

Figure 9 and Figure 10 show the relative velocity vectors and the static pressure distributions around the blades of RRtype in respective blade row distances $L=10,30 \mathrm{~mm}$ at the radial position $r / r_{c}=0.75$. The relative angle of the front and rear rotors is $\theta_{f r}=0^{\circ}$ as a representative. In Figure 9 and Figure 10, the blade indicated with the black diagonal lines is a subject in Figure 7 and Figure 8. The large separation and stall regions could be observed on the blade surface of the front rotor for $L=10 \mathrm{~mm}$ in Figure 9(a) although these regions were diminished for $L=30 \mathrm{~mm}$ in Figure 9(b). The large separation regions also occurred near the leading edge of the front rotor blades on the suction surface for $L=10 \mathrm{~mm}$ in Figure 9(a), however, the separation regions diminished for $L=30$ 


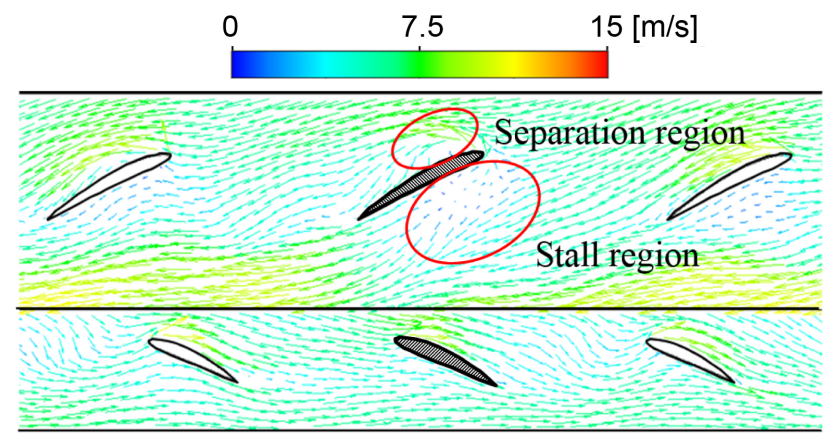

(a)

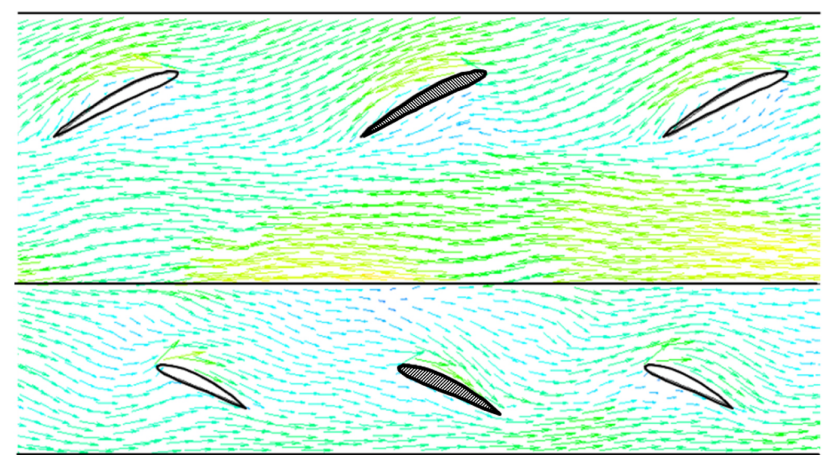

(b)

Figure 9. Relative velocity vectors at $r / r_{c}=0.75\left(0.1 Q_{d} \theta_{f r}=0^{\circ}\right)$. (a) $L=10 \mathrm{~mm}$; (b) $L=30 \mathrm{~mm}$.

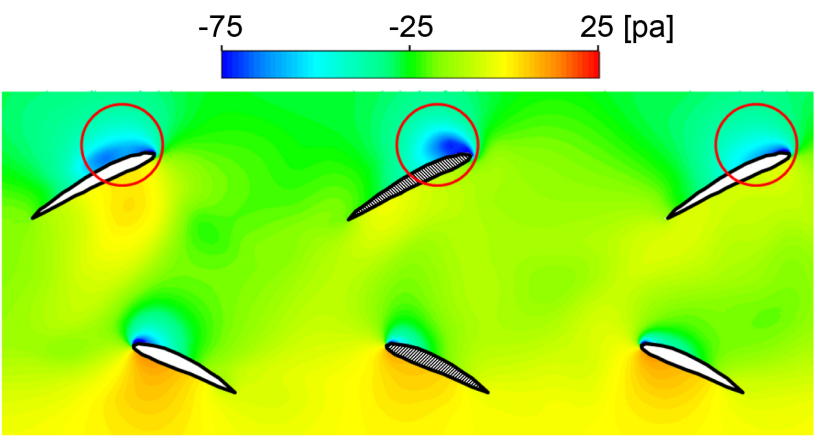

(a)

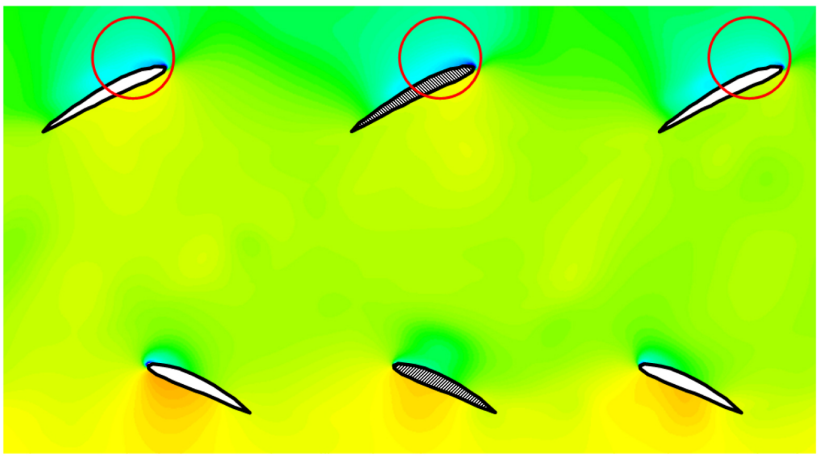

(b)

Figure 10. Static pressure distributions at $r / r_{c}=0.75\left(0.1 Q_{d} \theta_{f r}=\right.$ $0^{\circ}$ ). (a) $L=10 \mathrm{~mm}$; (b) $L=30 \mathrm{~mm}$. 
$\mathrm{mm}$ in Figure 9(b). Furthermore, the pressure distribution between the front and rear rotors in the respective blade row distances $L=10,30 \mathrm{~mm}$ was focused. In both of the blade row distance $L=10 \mathrm{~mm}$ and $L=30 \mathrm{~mm}$, the constitution of the complex and non-uniform pressure field was observed obviously from the trailing edge of the front rotor to the leading edge of the rear rotor. In the case of $L=10 \mathrm{~mm}$, the pressure field of the front rotor blades on the pressure side interferes strongly with the pressure field of the rear rotor blades on the suction side compared with the case of $L=30 \mathrm{~mm}$. Furthermore, the axial and circumferential components of the absolute velocity fluctuations at $r / r_{c}=0.75$ for $L=10$ $\mathrm{mm}$ and $L=30 \mathrm{~mm}$ are shown in Figure 11 and Figure 12. The vertical axis is the axial and circumferential components of the absolute velocity fluctuations $\Delta V_{a}, \Delta V_{c}$ and the horizontal axis is the relative angle of the front and rear rotors $\theta_{f r}$ in Figure 11 and Figure 12. The circumference component $\Delta V_{c}$ is a positive in the direction of the front rotor rotation. In Figure 11 and Figure 12, the axial and circumferential velocity fluctuated with the variation of the relative angle of
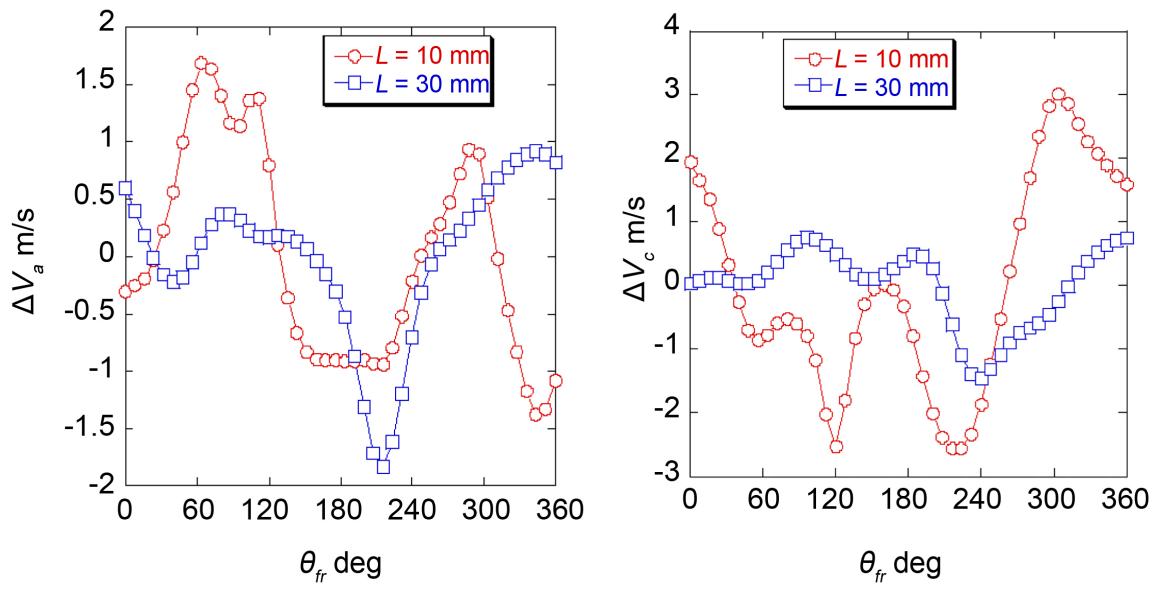

Figure 11. Absolute velocity fluctuations $3 \mathrm{~mm}$ downstream from the trailing edge of the front rotor $\left(0.1 Q_{\phi} r / r_{c}=0.75\right)$.
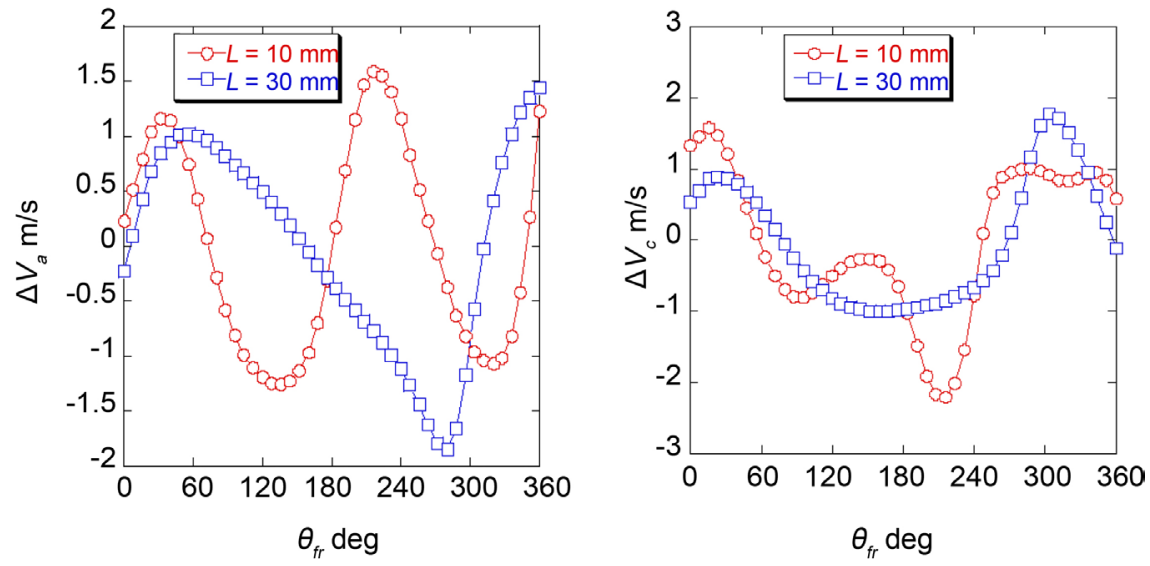

Figure 12. Absolute velocity fluctuations $3 \mathrm{~mm}$ upstream from the leading edge of the rear rotor $\left(0.1 Q_{\phi} r / r_{c}=0.75\right)$. 
the front and rear rotors $\theta_{f r}$, and the influence on the flow field of the front rotor by the rear rotor (i.e. the back flow) and the strong interference by the front rotor are observed for both $L=10 \mathrm{~mm}$ and $L=30 \mathrm{~mm}$ at the low flow rate $0.1 Q_{d}$. Figure 13 shows the meridional velocity vectors on a vertical plane of RRtype at the low flow rate $0.1 Q_{d}$ in respective blade row distances $L=10,30 \mathrm{~mm}$. The rotational direction of the front rotor is the back side of the paper and that of the rear rotor is the front side of the paper. There are some vortexes between the front and rear rotors and near the blades of each rotor for both $L=10 \mathrm{~mm}$ and $L=$ $30 \mathrm{~mm}$. The strong back flow associated with the tip leakage flow was observed for both $L=10 \mathrm{~mm}$ and $L=30 \mathrm{~mm}$ in Figure 13. These vortex regions cause the irregularity of the wake and the potential interference of RRtype. In the blade row distance $L=10 \mathrm{~mm}$, the distance between the vortex centers is close and the vortexes more influence each other than the blade row distance $L=30 \mathrm{~mm}$.

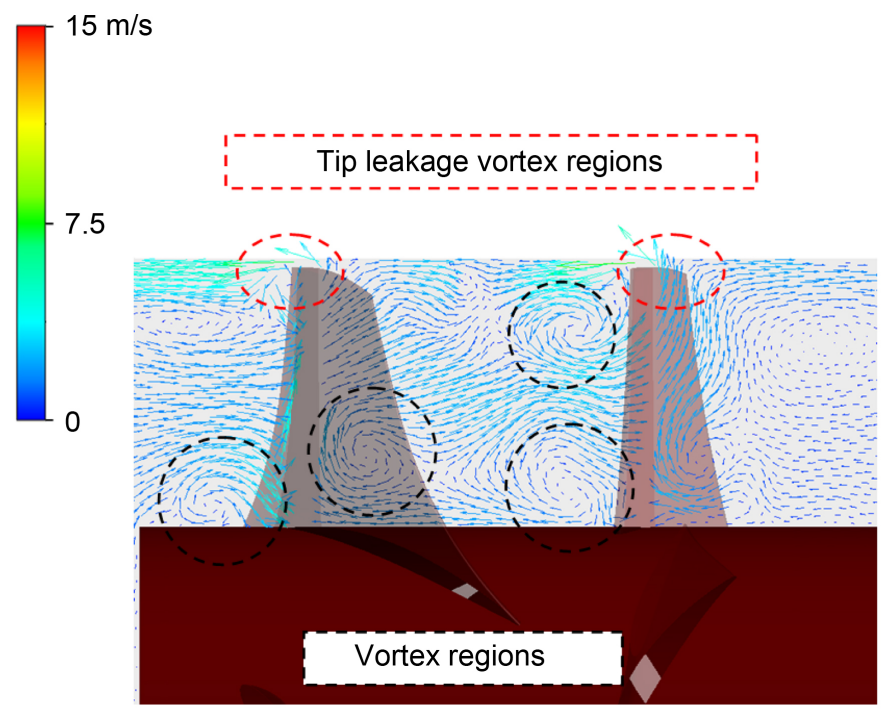

(a)

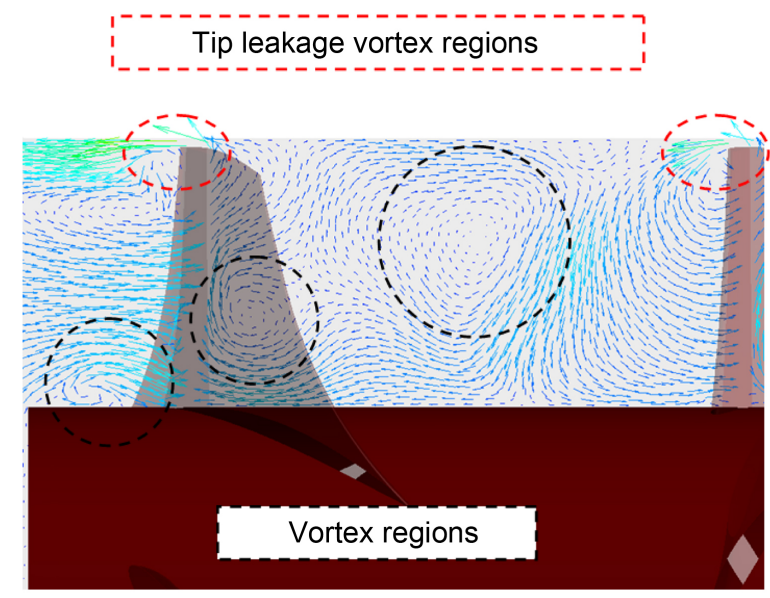

(b)

Figure 13. Meridional velocity vectors $\left(0.1 Q_{\phi} \theta_{f r}=0^{\circ}\right.$ ). (a) $L=10$ $\mathrm{mm}$; (b) $L=30 \mathrm{~mm}$. 
From the above, these strong potential and wake interference between the front and rear rotors lead to the large separation and stall regions around the front rotor blades. In the case of $L=10 \mathrm{~mm}$, it is considered that the fan static pressure and static pressure efficiency were not higher than that of $L=30 \mathrm{~mm}$ at the low flow rate $0.1 Q_{d}$ because of the strong and irregular wake and potential interference in association with the decrease of the blade row distance.

\section{Conclusion}

The contra-rotating small-sized axial fan was designed and the performance, wake and potential interference between the front and rear rotors were investigated at the low flow rate $0.1 Q_{d}$ with the unsteady numerical analysis. The fan static pressure of $L=10 \mathrm{~mm}\left(P_{s}=11.47 \mathrm{~Pa}\right)$ was larger than that of $L=30 \mathrm{~mm}$ $\left(P_{s}=11.45 \mathrm{~Pa}\right)$ at the design flow rate $Q_{d}=0.016 \mathrm{~m}^{3} / \mathrm{s}$ in the experiment. On the other hand, the fan static pressure of $L=10 \mathrm{~mm}\left(P_{s}=26.87 \mathrm{~Pa}\right)$ was smaller than that of $L=30 \mathrm{~mm}\left(P_{s}=27.50 \mathrm{~Pa}\right)$ at the low flow rate $0.1 Q_{d}$. The influence of the blade row distance in the range of $L=10-30 \mathrm{~mm}$ on the fan static pressure was small, but there is a tendency that the fan static pressure of $L=30 \mathrm{~mm}$ became large compared to that of $L=10 \mathrm{~mm}$ at the low flow rate. This characteristic could be confirmed in the numerical analysis results too. Pressure fields of the trailing edge of the front rotor and the leading edge of the rear rotor at the low flow rate $0.1 Q_{d}$ fluctuate irregularly at each radial position. These results lead to the thinking that the flow condition and the potential interference between the front and rear rotors are significantly complex. The strong potential and wake interference between the front and rear rotors lead to the separation and stall regions around the front rotor blades. In the case of $L=10 \mathrm{~mm}$, the fan static pressure and static pressure efficiency were not higher than those of $L=30 \mathrm{~mm}$ at the low flow rate $0.1 Q_{d}$ because of the strong and irregular wake and potential interference in association with the decrease of the blade row distance. The detailed performance and internal flow in wide flow rate range of the contra-rotating small sized axial fan were clarified from our research. Therefore, the high performance design of the contra-rotating small size fan needs to be investigated using the results of the internal flow condition as the future work.

\section{Acknowledgements}

The authors wish to show our special thanks to the supports by the Komiya research aid, the project research aid from Tokushima University and Japan Science and Technology Agency.

\section{References}

[1] Furukawa, A., Shigemitsu, T. and Watanabe, S. (2007) Performance Test and Flow Measurement of Contra-Rotating Axial Flow Pump. Journal of Thermal Science, 16, 7-13. https://doi.org/10.1007/s11630-007-0007-4

[2] Furukawa, A., Cao, Y., Okuma, K. and Watanabe, S. (2000) Experimental Study of Pump Characteristics of Contra-Rotating Axial Flow Pump. Turbomachinery, 67, 
245-252.

[3] Nouri, H., Ravelet, F., Bakir, F., Sarraf, C. and Rey, R. (2012) Design and Experimental Validation of a Ducted Counter-Rotating Axial-Flow Fans System. Journal of Fluids Engineering, 134, Article ID: 104504. https://doi.org/10.1115/1.4007591

[4] Shigemitsu, T., Furukawa, A., Okuma, K. and Watanabe, S. (2002) Experimental Study on Rear Rotor Design in Contra-Rotating Axial Flow Pump. Proceedings of 5th JSME/KSME Fluids Engineering Conference, Nagoya, 17-21 November 2002, 1453-1548.

[5] Wang, C. and Huang, L. (2014) Passive Noise Reduction for a Contrarotating Fan. Journal of Turbomachinery, 137, Article ID: 031007.

https://doi.org/10.1115/1.4028357

[6] Sanders, A.J., Papalia, J. and Fleeter, S. (2002) Multi-Blade Row Interactions in a Transonic Axial Compressor: Part I-Stator Particle Image Velocimetry (PIV) Investigation. Journal of Turbomachinery, 124, 10-18.

https://doi.org/10.1115/1.1411973

[7] Nouri, H., Danlos, A., Ravelet, F., Bakir, F. and Sarraf, C. (2013) Experimental Study of the Instationary Flow between Two Ducted Counter-Rotating Rotors. Journal of Engineering for Gas Turbines and Power, 135, Article ID: 022601. https://doi.org/10.1115/1.4007756

[8] Shigemitsu, T., Fukutomi, J. and Okabe, Y. (2010) Performance and Flow Condition of Small-Sized Axial Fan and Adoption of Contra-Rotating Rotors. Journal of Thermal Science, 19, 1-6. https://doi.org/10.1007/s11630-010-0001-0

[9] Shigemitsu, T., Fukuda, H. and Hirosawa, K. (2017) Unsteady Flow Condition between Front and Rear Rotor of Contra-Rotating Small Sized Axial Fan. Open Journal of Fluid Dynamics, 7, 371-385. https://doi.org/10.4236/ojfd.2017.73025

[10] Liu, S., Huang, R. and Lin, C. (2010) Computational and Experimental Investigations of Performance Curve of an Axial Flow Fan Using Downstream Flow Resistance Method. Experimental Thermal and Fluid Science, 34, 827-837. https://doi.org/10.1016/j.expthermflusci.2010.01.011

[11] Jin, G., Ouyang, H. and Du, Z. (2013) Experimental Investigation of Unsteady Flow in Axial Skewed Fans According to Flow Rates. Experimental Thermal and Fluid Science, 48, 81-96. https://doi.org/10.1016/j.expthermflusci.2013.02.011

[12] Shigemitsu, T., Fukutomi, J., Okabe, Y. and Iuchi, K. (2010) Performance and Flow Condition of Contra-Rotating Small-Sized Axial Fan at Partial Flow Rate. International Journal of Fluid Machinery and Systems, 3, 271-278. https://doi.org/10.5293/IJFMS.2010.3.4.271

[13] Ito, T., Minorikawa, G., Nagamatsu, A. and Suzuki, S. (2006) Experimental Research for Performance and Noise of Small Axial Flow Fan (Influence of Parameter of Blade). Transactions of the JSME, 72, 670-667. (In Japanese)

https://doi.org/10.1299/kikaib.72.670 